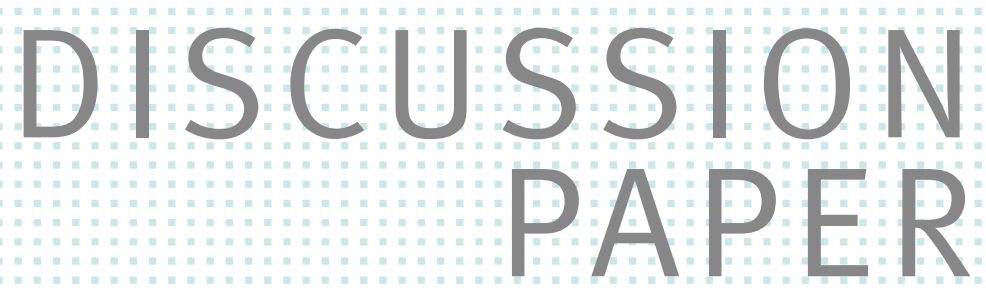

// OLIVIER BOS AND MARTIN POLLRICH

\title{
Optimal Auctions with Signaling Bidders
}




\title{
Optimal Auctions with Signaling Bidders*
}

\author{
Olivier Bos ${ }^{\dagger}$ and Martin PollRich ${ }^{\ddagger}$
}

October 6, 2020

\begin{abstract}
We study optimal auctions in a symmetric private values setting, where bidders' care about winning the object and a receiver's inference about their type. We reestablish revenue equivalence when bidders' signaling concerns are linear, and the auction makes participation observable via an entry fee. With convex signaling concerns, optimal auctions are fully transparent: every standard auction, which reveals all bids yields maximal revenue. With concave signaling concerns there is no general revenue ranking. We highlight a trade-off between maximizing revenue derived from signaling, and extracting information from bidders. Our methodology combines tools from mechanism design with tools from Bayesian persuasion.

Keywords: optimal auctions, revenue equivalence, Bayesian persuasion, information design

JEL classification: D44; D82
\end{abstract}

${ }^{*}$ We are grateful to participants at the $1^{\text {st }}$ KIT-Paris2-ZEW Workshop on Market Design, the 2019 INFORMS Annual Meeting and the $4^{\text {th }}$ Internal Conference of the CRC TR 224 for their helpful comments. Financial support from the ANR SIGNAL (ANR-19-CE26-0009) is gratefully acknowledged. This research has been conducted as part of the Labex MME-DII (ANR11-LBX-0023-01) project. Martin Pollrich gratefully acknowledges financial support from the Deutsche Forschungsgemeinschaft through CRC TR 224 (Project B01).

†Panthéon-Assas University (LEMMA), Institut Universitaire de France (IUF) and ZEW-Leibniz Centre for European Economic Research. E-mail: olivier.bos@u-paris2.fr.

†University of Bonn. E-mail: martin.pollrich@uni-bonn.de. 


\section{Introduction}

Since 1945, the Hospices de Beaune ${ }^{1}$, in Burgundy (France), organizes an annual wine auction to raise money for local retirement houses and hospitals. In a special segmentthe "pièce des Présidents" — some wine is auctioned to raise money for special charity purposes. This segment attracts special attention not the least due to the involvement of celebrities. In the 2017 "pièce des Présidents" auction two barrels of Corton Clos du Roi Grand Cru were sold at a total price of $€ 410,000$. During the regular auction, the same wine realized prices ranging from $€ 30,000$ to $€ 40,000$ per barrel. Roughly speaking, public attention increased the price per barrel by $500 \% .^{2}$

This is but one example of an auction where bidders have signaling concerns, i.e., bidders care about the object at sale but also about how they are perceived by others. Mandel (2009) identifies signaling as an important aspect for buying and investing in artwork. Signaling incentives arise for several reasons in takeover bidding: (i) competing firms issue equity or debt for financing (Liu, 2012), and (ii) compensation or career prospects of the bidders' managers depend on uninformed investors perception of their value (Giovannoni and Makris, 2014). In a procurement context, a good performance in one auction signals high average efficiency and thereby raises the prospects of being qualified for future tenders (Wan and Beil, 2009).

In this manuscript we study auction design when bidders care about the information conveyed through their own performance. How others perceive the bidders' performance crucially depends on the auction design. For example, in a first-price auction, outsiders observe the winner's bid (via the price) but no other bids. This allows precise inference on the winner's type, but only noisy inference on losers' types. In a second-price auction outsiders observe the highest losing bid, hence inference on all bidders remains noisy. With this in mind, the design of the auction affects the possibilities for outside inference, which in turn affects bidding behavior and auction revenue. Our analysis is then looking for the revenue-maximizing auction design when bidders have signaling concerns.

In their seminal contributions Myerson (1981) and Riley and Samuelson (1981) show that - absent signaling concerns - every standard auction yields the same revenue. This needs no longer to be the case when bidders care for signaling. ${ }^{3}$ We study an auction environment with independent private values. In addition, bidders preferences depend

\footnotetext{
${ }^{1}$ https://www.beaune-tourism.com/discover/hospices-de-beaune-wine-auction

${ }^{2}$ Similar patterns arose in the previous years. Data for 2016 and 2017 are available at http://hospices-de-beaune.com/index.php?/hospicesdebeaune/content/download/3869/14085/ version/1/file/catalogue_resultats_2016.pdf and http://hospices-de-beaune.com/index. $\mathrm{php}$ ?/hospicesdebeaune/content/download/4248/15476/version/1/file/Vente+des+vins+-+ Catalogue+des $+r \%$ C3\%A9sultats +2017 .pdf

${ }^{3}$ Giovannoni and Makris (2014), Bos and Truyts (2019) study environments where different auction formats (and bid disclosure policies) yield different auction revenue. Goeree (2003), Molnar and Virag (2008), Katzman and Rhodes-Kropf (2008) find revenue equivalence in their respective settings.
} 
on the mean of posterior beliefs about their own type. These posteriors are formed conditional on the outcome of the auction: the winner's identity and each bidder's payment. Our main result lies in a decomposition of the auctioneer's revenue into the standard revenue from the auction without signaling concern, and a signaling component. The latter consisting of the bidders' total signaling value, minus the expected signaling value of a non-participating bidder (which represents an endogenous outside option). Whether revenue equivalence obtains depends on the curvature of the signaling motive in a bidder's utility (which affects total signaling value), and the observability of bidders' participation decisions (e.g., via charging entry fees), which affect the outside option.

When signaling concerns are linear (i.e. mean posteriors enter linearly in bidders' utility) revenue equivalence obtains between standard auctions that use entry fees. Under linearity the signaling value is independent of the auction format, hence only the endogenous outside option yields differences in revenue. The auctioneer uses entry fees to extract all signaling value from bidders, independent of whether they end up winning the object.

Matters are different when the signaling concerns is not linear. When bidders' preferences are convex, revenue increases in the amount of information the auction reveals. An all-pay auction yields maximal revenue, because in the fully separating equilibrium a bidder's payment perfectly reveals her type (maximizing signaling value) and all bidders pay their bid (extracting signaling value). Moreover, every standard auction that additionally reveals all bids yields the same revenue, because revealing bids automatically reveals whether a bidder participated.

With concave signaling concerns a general revenue ranking cannot be established. The information revealed during the auction affects revenue in two ways. First, revealing less information about bidders' types increases signaling value, which in turn increases revenue via more aggressive bidding. Second, to extract all signaling value the auctioneer should again charge an entry fee, which, however, reveals additional information about bidders and thereby reduces signaling value. Therefore, if participation is already fully observable it becomes optimal to reveal only the winner's identity. Moreover, if participation in the auction is high enough, charging an entry fee is optimal, because the reduction in signaling value is small.

Ex-post payments play a crucial role in our analysis, because beliefs are formed based on the realized auction outcome. This prevents us from directly applying standard tools from auction theory (e.g., Myerson, 1981), that use interim payments. We adapt methods from Bayesian persuasion to work with distributions over posterior beliefs. This intermediate step allows us to move the entire analysis to the interim stage.

Auctions with signaling concerns have been recently investigated by Giovannoni and 
Makris (2014) and Bos and Truyts (2019). ${ }^{4}$ The former consider auctions that reveal the winner's identity together with four disclosure policies: no information, the highest bid, the second highest bid, or all bids (each together with the respective bidder's identity). In particular, only a (strict) subset of bidders' payments is observable. Bos and Truyts compare second-price and English auctions, that reveal the winner's identity and her payment. Both studies compare specific auction formats and disclosure policies, and establish a failure of revenue equivalence. Our analysis, which covers all standard auctions, provides conditions under which revenue equivalence is restored via the use of an entry fee and optimal bid disclosure.

Our paper is also related to the literature on mechanism design with aftermarkets. Calzolari and Pavan $(2006 a, b)$ study contracting environments where the agent participates in an aftermarket. They find conditions under which no information release to the aftermarket is optimal. Dworczak (2020) analyzes an auction environment with a very general aftermarket. He restricts the analysis to cut-off mechanisms in which the information revealed about the winner only depends on the losers' bids. These mechanisms rule out disclosure of information contained only in the winner's bid, such as the price in a first-price auction or the entire vector of payments in the all-pay auction, which we show is optimal in some cases. Also Molnar and Virag (2008) study auctions with an aftermarket, to which only the winner is concerned by signaling (and there is no aftermarket if there is no winner). They show that it is optimal to reveal (conceal) the winner's type when the signaling incentive is convex (concave). In our setting all bidders care about how they are perceived, irrespective of whether they win. This brings about a novel decomposition of revenue into the standard non-signaling component and a signaling component, which can be analyzed using methods from information design. In addition, a new trade off arises from the bidders' endogenous outside option, implicitly given by the signaling value from abstention, which yields new insights in the concave and convex cases.

Information disclosure in auctions has first been analyzed in the setting of affiliated values by Milgrom and Weber (1982). Mechanism design problems with allocative and informational externalities have also been studied by Jehiel and Moldovanu (2000, 2001). The underlying assumption in this strand of literature is that an agent's valuation depends also on other agents' private information (and allocation). In our setting a bidder's utility is affected by the aftermarket's belief about her own valuation, while such beliefs have no impact in the literature on mechanism design with interdependent valuations.

The paper is organized as follows. Section 2 introduces the formal setting. In Section 3 we propose a decomposition of the seller's revenue to identify the play of non-signaling

\footnotetext{
${ }^{4}$ There are also contributions about information transmission comparing specific auction formats followed by oligopoly competition. See, e.g., Goeree (2003), Das Varma (2003), Katzman and RhodesKropf (2008) and von Scarpatetti and Wasser (2010).
} 
and signaling components. This preliminary result defines the outline of our analysis. Section 4 studies the case of linear signaling concerns. In Section 5 we derive optimal auctions when the signaling concerns are convex, and in Section 6 analyzes the concave case. We conclude and discuss our results in Section 7.

\section{Formal Setting}

We consider $n$ bidders, who bid for a single object in an auction, and also care about the inference of an outside observer about their type.

Bidder $i$ 's valuation for the object (her 'type'), is denoted $V_{i}$, and is assumed i.i.d. and drawn according to a distribution function $F$ with support on $[\underline{v}, \bar{v}] \subset \mathbb{R}_{+}$. Let $f \equiv F^{\prime}$ denote the density function, $G \equiv F^{n-1}$ the distribution function of the highest order statistic among $n-1$ remaining valuations and $g \equiv G^{\prime}$ the corresponding density function. Bidder $i$ 's realization of $V_{i}$, denoted $v_{i}$, is her private information, but the number of bidders and the distribution $F$ are common knowledge.

We consider standard auctions in which each bidder submits a (non-negative) bid $b_{i}$, the highest bidder wins (ties broken at random), and bidder $i$ 's payment $p_{i}$ depends on the entire vector of bids, i.e., $p_{i}\left(b_{1}, \ldots, b_{n}\right)$. In addition, the auctioneer controls participation, e.g., via charging an entry fee $\varphi$ or setting a reserve price $r$. Entry fees play a prominent role in our analysis. Under a non-zero entry fee a bidder who wishes to submit a bid first has to pay the entry fee. We do not allowed for extortionate fees, i.e., whenever the object is not allocated all bidders' payments are zero. ${ }^{5}$ When all payments are observable, an entry fee allows inference on participation, while a reserve price does not. With slight abuse of notation we denote $p_{i}\left(b_{1}, \ldots, b_{n}\right)$ the final payment that bidder $i$ makes, potentially including the entry fee. In particular, we may have $p_{i}>0$ even though bidder $i$ did not win the object.

Each bidder cares about winning the object, and about the inference of an outside observer, the 'receiver', about her type. This receiver can represent, e.g., the general public or press, business contacts or acquaintances of the bidder, or experts related to the object at sale. The receiver observes the outcome of the auction $\mathcal{O}=\left(i^{\star}, p_{1}, \ldots, p_{n}\right)$, where $i^{\star}$ is the winner's identity and $p_{i}$ the payment made by bidder $i$. We thus implicitly assume that the outcome $\mathcal{O}$ is public information. This assumption is met by the examples mentioned in the introduction: in the European Union a directive on public procurement specified the information to be included in a contract award notice, ${ }^{6}$ the EU directive on

\footnotetext{
${ }^{5}$ As in Lizzeri (1999) the auctioneer can extract all signaling value by charging a participation fee and threatening non-participant with the worst possible belief. Though theoretically possible, it is hard to justify such a fee in practice when sale may not take place.

${ }^{6}$ See Annex V part D of Directive 2014/24/EU of the European Parliament and of the Council of 26 February 2014 on public procurement: https://eur-lex.europa.eu/legal-content/EN/TXT/PDF/
} 
takeover bids lays down the information concerning bids, ${ }^{7}$, and in many art auctions that attract publicity (i.e., where signaling likely matters) the auction outcome is published in the press and the websites of the big auction houses. Many papers on signaling in auctions assume payments can be concealed, and only the winner's identity is public. Though such an assumption heavily facilitates the analysis, it is neither backed up by institutional practical (as suggested by the examples given above), nor does it look very convincing to arbitrarily decide which part of the allocation is public and which not.

Based on the auction outcome $\mathcal{O}$, the receiver forms a posterior belief about each bidder's type, denoted as $\mu_{i}(\mathcal{O})$. We assume that a bidder's utility depends on the receiver's belief only through the posterior mean, i.e., the expected value given the posterior distribution. ${ }^{8}$ This is a reasonable assumption for instance in the context of takeover bidding, where a bidder's utility is affected by the decision of a competitive aftermarket of riskneutral investors/firms (e.g., Liu, 2012, Giovannoni and Makris, 2014). ${ }^{9}$ Formally, there is an increasing function $\Phi:[\underline{v}, \bar{v}] \rightarrow \mathbb{R}_{+}$, such that the bidder's utility is given by

$$
u_{i}\left(v_{i}, \mathcal{O}\right)=\left\{\begin{aligned}
v_{i}-p_{i}+\Phi\left(\mathbb{E}\left(V_{i} \mid \mathcal{O}\right)\right), & \text { if } i=i^{\star}, \\
-p_{i}+\Phi\left(\mathbb{E}\left(V_{i} \mid \mathcal{O}\right)\right), & \text { if } i \neq i^{\star}
\end{aligned}\right.
$$

The function $\Phi$ represents a reduced form of a (continuation) game in which the receiver chooses an action that directly affects the bidder's payoff. Note that a bidder's utility is not affected by the receiver's belief about other bidders' types. For instance, from an individual bidder's perspective it is equivalent to have either a different or the same receiver for each bidder.

Any standard auction defines a signaling game among bidders and the receiver. We consider symmetric perfect Bayesian equilibrium, consisting of the bidders' bidding strategies $\beta:[\underline{v}, \bar{v}] \rightarrow \mathbb{R}_{+}$and the receiver's belief $\left(\mu_{1}, \ldots, \mu_{n}\right) .{ }^{10}$ Each bidder's bidding strategy is optimal, given the other bidders' bidding and the receiver's beliefs. Also, the receiver's beliefs are Bayesian consistent with the bidding strategy. In our analysis we focus on equilibria in which bidders use strictly increasing bidding functions. This assumption is in line with the usual focus in auction theory. I thus allows for a straightforward com-

?uri=CELEX : 02014L0024-20200101\&from=EN (last accessed October 6th 2020).

${ }^{7}$ See Article 6 of Directive 2004/25/EC of the European Parliament and of the Council of 21 April 2004 on takeover bids: https ://eur-lex. europa.eu/legal-content/EN/TXT/PDF/?uri=CELEX: 32004L0025\&from=EN (last accessed October 6th 2020).

${ }^{8}$ Note that we do not assume that a bidder's type $v_{i}$ directly affects the receiver's payoff. The receiver cares about some other characteristic of the bidder, which is correlated with the bidder's type. See also the example at the end of this section.

${ }^{9}$ Similar assumptions are made the context of Bayesian Persuasion (e.g., Dworczak and Martini, 2019, Rayo and Segal, 2010). An interpretation given there is that a single receiver takes an action, and the receiver preferences are, e.g., symmetric and single peaked, such that the decision equals the posterior mean.

${ }^{10}$ To save on notation we do not formalize the bidders' entry decision. Under an effective entry fee each bidder first decides whether to enter the auction. The receiver observes whether a bidder paid $\varphi$, and updates her belief accordingly. 
parison with the no-signaling benchmark. In the respective sections we briefly discuss whether the this focus is restrictive. In the language of signaling games, we thus focus on separating equilibria, and do not emphasize the multiplicity of equilibria, selection and refinements. As a byproduct we show existence of such equilibria for the auctions and cases under consideration.

We conclude this section with an illustrative example for the bidders' utility functions. Suppose the bidder's valuation $v$ is uniformly distributed on $[0,1]$. The receiver cares about the bidder's characteristic $\theta$, given by $\theta=\alpha v+(1-\alpha) s$, where $s$ is uniformly distributed on $[0,1]$, independent of $v$, and $\alpha \in(0,1)$. The receiver takes an action $a$ to maximize her utility $U_{R}(a, \theta)=-(a-\theta)^{2}$. Given the auction outcome $\mathcal{O}$, the receiver chooses $a=\mathbb{E}(\theta \mid \mathcal{O})$. We have that

$$
a=\mathbb{E}(\theta \mid \mathcal{O})=\mathbb{E}(\alpha v+(1-\alpha) s \mid \mathcal{O})=\alpha \mathbb{E}(V \mid \mathcal{O})+\frac{1-\alpha}{2}
$$

If $\Psi(a)$ describes the impact of the receiver's action on the bidder's utility, we can define $\Phi(\mathbb{E}(V \mid \mathcal{O}))=\Psi\left(\alpha \mathbb{E}(V \mid \mathcal{O})+\frac{1-\alpha}{2}\right)$. The shape of $\Psi$ determines the shape of $\Phi$. In the context of our introductory example of the Hospices de Beaune, $\theta$ may represent a bidder's altruism. Our model assumes that a bidder's altruism is correlated with her valuation for the wine auctioned, where $\alpha$ measures the degree of correlation.

\section{Payoff-(non)-equivalence}

The aim of this section is to establish a decomposition of the the seller's revenue. We show that the revenue is the sum of a non-signaling component, which corresponds to the respective auction revenue without signaling concerns, and a signaling component. Such a decomposition implies that all differences in auction revenue stem from the second component, because the first component - the standard revenue - is the same across all auction formats.

In the following we expand standard arguments from auction theory to our setting with signaling bidders (Riley and Samuelson, 1981, Krishna, 2009). Fix some auction format $A$ and suppose there is an equilibrium in which (i) bidder $i$ participates if and only if $v_{i} \geq \tau$, and (ii) (participating) bidders follow the strictly increasing bidding strategy $\beta^{A}:[\tau, \bar{v}] \rightarrow \mathbb{R}$. Denote $m^{A}(v)$ the expected payment and $\mathcal{W}_{\tau}^{A}(v)$ the expected value from signaling of a bidder with valuation $v \geq \tau$. Non-participating bidders, i.e., bidders with $v<\tau$, have $m^{A}(v) \equiv 0$ and $\mathcal{W}_{\tau}^{A}(v) \equiv \mathcal{W}_{\tau, 0}^{A}$. The value $\mathcal{W}_{\tau, 0}^{A}$ denotes the (interim) expected signaling value of a bidder who abstains from participation.

Consider a bidder with valuation $v \geq \tau$. His expected payoff from mimicking type $\widetilde{v} \geq \tau$ is

$$
\Pi(v, \widetilde{v})=G(\tilde{v}) v-m^{A}(\tilde{v})+\mathcal{W}_{\tau}^{A}(\tilde{v})
$$


At equilibrium the bidder's payoff is $\Pi(v):=\Pi(v, v)$ and using the envelope theorem ${ }^{11}$ it follows that

$$
G(v) v-m^{A}(v)+\mathcal{W}_{\tau}^{A}(v)=\Pi(v)=\Pi(\tau)+\int_{\tau}^{v} G(x) \mathrm{d} x
$$

A bidder with valuation $\tau$ is indifferent whether to participate, if

$$
\Pi(\tau)=\mathcal{W}_{\tau, 0}^{A}
$$

Using (2) and (3) we express the interim expected payment of a bidder as follows

$$
m^{A}(v)=G(v) v-\int_{\tau}^{v} G(x) \mathrm{d} x+\mathcal{W}_{\tau}^{A}(v)-\mathcal{W}_{\tau, 0}^{A}=G(\tau) \tau+\int_{\tau}^{v} g(x) x \mathrm{~d} x+\mathcal{W}_{\tau}^{A}(v)-\mathcal{W}_{\tau, 0}^{A}
$$

Note that this expected payment depends on the auction format only via the signaling component $\mathcal{W}_{\tau}^{A}(v)-\mathcal{W}_{\tau, 0}^{A}$. The auctioneer's revenue is $n \int_{\underline{v}}^{\bar{v}} m^{A}(v) \mathrm{d} F(v)=n \int_{\tau}^{\bar{v}} m^{A}(v) \mathrm{d} F(v)$. From the existing literature (e.g., Riley and Samuelson (1981)) we get that

$$
n \int_{\tau}^{\bar{v}}\left(G(\tau) \tau+\int_{\tau}^{v} g(x) x \mathrm{~d} x\right) \mathrm{d} F(v)=\operatorname{Rev}^{\mathcal{M}}(\tau)
$$

where $\operatorname{Rev}^{\mathcal{M}}(\tau)$ is the revenue in the auction without signaling concern. Regarding the second term in 4, capturing the bidders' signaling values, recall that bidders preferences only depend on posterior means. Denote by $H_{\tau}^{A}$ the distribution over posterior means, that is induced by the equilibrium of the auction. ${ }^{12}$ In particular, we have that $\int_{\underline{v}}^{\bar{v}} v \mathrm{~d} H_{\tau}^{A}(v)=\int_{\underline{v}}^{\bar{v}} v \mathrm{~d} F(v)$. As in the literature on Bayesian persuasion (e.g., Dworczak and Martini, 2019), we can rewrite a bidder's signaling value using the distribution over posterior means, instead of the interim values $\mathcal{W}_{\tau}^{A}$. Formally, we have that

$$
\begin{aligned}
\int_{\tau}^{\bar{v}}\left(\mathcal{W}_{\tau}^{A}(v)-\mathcal{W}_{\tau, 0}^{A}\right) \mathrm{d} F(v) & =F(\tau) \mathcal{W}_{\tau, 0}^{A}+\int_{\tau}^{\bar{v}} \mathcal{W}_{\tau}^{A}(v) \mathrm{d} F(v)-\mathcal{W}_{\tau, 0}^{A} \\
& =\int_{\underline{v}}^{\bar{v}} \Phi(v) \mathrm{d} H_{\tau}^{A}(v)-\mathcal{W}_{\tau, 0}^{A}
\end{aligned}
$$

Combining these steps yields the following Proposition.

Proposition 1. Consider a standard auction A, in which every bidder follows a strictly increasing bidding strategy, and participates whenever his type is above $\tau$. The revenue

\footnotetext{
${ }^{11}$ See Milgrom and Segal (2002). The objective in $(1)$ is differentiable in $v$, and the derivative $(G(\widetilde{v}))$ is uniformly bounded.

${ }^{12}$ The distribution $H_{\tau}^{A}$ depends on the auction format, via the induced information disclosure, and on the participation threshold $\tau$, because all types $v<\tau$ are lumped into the same information which effects the resulting posterior mean.
} 
in this auction is given by

$$
\operatorname{Rev}^{\mathcal{M}}(\tau)+n\left(\int_{\underline{v}}^{\bar{v}} \Phi(v) \mathrm{d} H_{\tau}^{A}(v)-\mathcal{W}_{\tau, 0}^{A}\right)
$$

It is immediate from Proposition 1 that differences in auction revenue are solely due to the signaling component, i.e., due to differences in the distribution over posterior means $H_{\tau}^{A}$ and the signaling value to non-participating bidders $\mathcal{W}_{\tau, 0}^{A}$. The auction extracts the bidders' signaling value, given by the term $\int_{\underline{v}}^{\bar{v}} \Phi(v) \mathrm{d} H_{\tau}^{A}(v)$. But signaling creates an endogenous outside option, given by $\mathcal{W}_{\tau, 0}^{A}$, which clearly cannot be extracted. Auction design thus corresponds to maximizing the bidders' signaling value - effectively performing information design - while keeping the outside option low. In the following we explore the optimal auction design in the light of formula (5) for linear, convex and concave signaling concerns.

Remark 1. The decomposition of the auction revenue in a signaling and a non-signaling component does not rely on our assumption that bidders care only about the posterior mean. For any preference that is additively separable and where signaling preferences are independent of the bidders' true type we get a revenue decomposition as in (5). However, restricting to preferences over posterior means greatly simplifies the following analysis.

\section{Linear Signaling Concerns}

In this section, we consider a linear inference $\Phi(v)=\lambda v$, with $\lambda>0$ the strength of a bidder's signaling concerns. Previous results indicate that even with linear inference revenue equivalence may fail. We show that revenue equivalence holds when the auctioneer uses an entry fee to make public an individual bidder's decision whether to enter the auction.

The next Lemma is an immediate consequence of Proposition 1.

Lemma 1. Consider a standard auction, in which every bidder follows a strictly increasing bidding strategy, and participates whenever his type is above $\tau$. The revenue in this auction is given by

$$
\operatorname{Rev}^{\mathcal{M}}(\tau)+n\left(\lambda \mathbb{E}(V)-\mathcal{W}_{\tau, 0}^{A}\right)
$$

Proof. From Proposition 1 we have that the revenue equals (5). Furthermore, we have that

$$
\int_{\underline{v}}^{\bar{v}} \Phi(v) \mathrm{d} H_{\tau}^{A}(v)=\lambda \int_{\underline{v}}^{\bar{v}} v \mathrm{~d} H_{\tau}^{A}(v)=\lambda \int_{\underline{v}}^{\bar{v}} v \mathrm{~d} F(v)=\lambda \mathbb{E}(V),
$$

where the middle equality uses the fact the induced distribution over posterior means preserves the mean of the original distribution $F$. 
With linear signaling concerns the bidders' signaling value does not depend on the auction format. As in the literature on Bayesian persuasion, any information structure is equally valuable for a risk-neutral sender. So why does auction revenue still differ when bidders signaling concern is linear? The bidders' outside option $\mathcal{W}_{\tau, 0}^{A}$ still depends on the auction design. A non-participating bidder always makes a zero payment and does not receive the object. But also participating bidders may end up without the object and making a zero payment, for instance all losing bidders in a first-price auction. The inability of an outside observer to tell apart losing bidders from non-participating bidders inflates the value $\mathcal{W}_{\tau, 0}^{A}$ and thereby reduces auction revenue.

Note that the value $\mathcal{W}_{\tau, 0}^{A}$ already differs between first and second-price auctions. In a first-price auction we infer that all losers have a type below the winner's, which is perfectly revealed from his payment. In a second-price auction the winner's payment reveals the second-highest type, and a loser has either exactly this type or a lower type. In many other auction formats it is impossible to tell apart a losing bidder who does not make any payment from a non-participating bidder. The auctioneer benefits from making an individual bidder's participation decision transparent, because this reduces the outside option $\mathcal{W}_{\tau, 0}^{A}$ to its minimum.

Proposition 2 (Revenue Equivalence). Consider a standard auction, in which every bidder follows a strictly increasing bidding strategy. When all types above $\tau$ participate and participation is observable (e.g. via an entry fee) the revenue equals

$$
\operatorname{Rev}^{\mathcal{M}}(\tau)+n \lambda(\mathbb{E}(V)-\mathbb{E}(V \mid V<\tau))
$$

Moreover, no other auction yields higher revenue.

Proof. Note that $\mathcal{W}_{\tau, 0}^{A} \geq \lambda \mathbb{E}(V \mid V<\tau)$, because by assumption all types below $\tau$ do not participate in the auction, hence never receive the object and pay zero. Furthermore, if participation is observable we get equality in the latter formula. Plugging this value into (6) yields (7).

A commonly applied way for making participation observable is to charge an entry fee. This way, an outsider who observes all payments made during the auction can tell apart losing bidders from non-participating bidders. Note that setting a reserve price does not yield maximal revenue, as the outsider cannot distinguish between participating losers and non-participating bidders, as long as individual bids are not fully disclosed.

Next we want to compare optimal levels of participation among situations with and without signaling concerns. We focus on auctions with entry fees, as these attain the maximal revenue determined in Proposition 2, with $\tau^{\star}(\lambda)$ the optimal level of participation under signaling strength $\lambda .{ }^{13}$ Note that $\tau^{\star}(0)=\tau^{\mathcal{M}}$, where $\tau^{\mathcal{M}}$ denotes the optimal

\footnotetext{
${ }^{13}$ In general there may not be a unique optimal level of participation. Our assumption of increasing
} 
participation cut-off in an auction without signaling concerns.

Corollary 1 (Optimal Participation). Assume virtual valuations are increasing. We have that

(i) $\tau^{\star}(\lambda) \leq \tau^{\star}\left(\lambda^{\prime}\right)<\tau^{\mathcal{M}}$ for all $\lambda>\lambda^{\prime}>0$.

(ii) There exists $\bar{\lambda}$ such that $\tau^{\star}(\lambda)=\underline{v}$, for all $\lambda>\bar{\lambda}$.

Proof. From Riley and Samuelson (1981) we know that

$$
\left(R e v^{\mathcal{M}}\right)^{\prime}(\tau)=n F^{n-1}(\tau)(1-F(\tau)-\tau f(\tau))=-n f(\tau) F^{n-1}(\tau)\left(\tau-\frac{1-F(\tau)}{f(\tau)}\right)
$$

Provided that virtual valuations $v-\frac{1-F(v)}{f(v)}$ are strictly monotone we have that $\operatorname{Rev}^{\mathcal{M}}(\tau)$ has a unique maximum $\tau^{\mathcal{M}}$. Furthermore, $\left(\operatorname{Rev}{ }^{\mathcal{M}}\right)^{\prime}(\tau)<0$ for all $\tau>\tau^{\mathcal{M}}$, and $\left(\operatorname{Re} v^{\mathcal{M}}\right)^{\prime}(\tau)>0$ for all $\tau \in\left(\underline{v}, \tau^{\mathcal{M}}\right)$. Together with the observation that $\mathbb{E}(V \mid V<\tau)$ strictly increases in $\tau,(i)$ follows. To prove (ii) note that the derivative of $\operatorname{Rev}^{\mathcal{M}}(\tau)$ is bounded. Hence, as soon as $\lambda$ becomes sufficiently large we have that $\lambda \frac{\partial}{\partial \tau} \mathbb{E}(V \mid V<\tau)>$ $\left(\operatorname{Re} v^{\mathcal{M}}\right)^{\prime}(\tau)$ for all $\tau>\underline{v}$ and thus full participation maximizes revenue in the auction with signaling.

We conclude this setting with an illustration of our results for the first- and the second-price auction.

Example 1 (Equilibrium of the first-price auction). Consider a first-price auction with entry fee $\varphi .^{14}$ The critical type $\tau$ pays the entry fee $\varphi$ and places a zero bid. Equations (3) and (4) together with $\beta(\tau)=0$ yields

$$
\beta(v)=\int_{\tau}^{v} \frac{x g(x)}{G(v)} d x+\frac{\mathcal{W}_{\tau}(v)-\mathcal{W}_{\tau}(\tau)}{G(v)}, \quad \forall v>\tau
$$

The signaling value $\mathcal{W}_{\tau}(v)$ can be expressed as follows

$$
\begin{aligned}
\mathcal{W}_{\tau}(v) & =\lambda G(v) v+(1-G(v)) \frac{\lambda}{1-G(v)} \int_{v}^{\bar{v}} \mathbb{E}(V \mid \tau<V<x) d G(x) \\
& =\lambda G(v) v+\int_{v}^{\bar{v}} \int_{\tau}^{x} \frac{\lambda y}{F(x)-F(\tau)} d F(y) d G(x) .
\end{aligned}
$$

Hence,

$$
\beta(v)=\int_{\tau}^{v} \frac{x g(x)}{G(v)} d x+\lambda \frac{G(v) v-G(\tau) \tau}{G(v)}+\int_{\tau}^{v} \int_{\tau}^{x} \frac{\lambda y}{F(x)-F(\tau)} d F(y) d G(x) .
$$

virtual valuations in Corollary 1 guarantees both existence and uniqueness of $\tau^{\star}(\lambda)$.

${ }^{14}$ The superscript $A$ is omitted in Example 1 and 2 , as they both focus on a specific auction format. 
Bos and Truyts (2018, Proposition 1) show that these bidding strategies indeed constitute an equilibrium of the first-price auction.

Example 2 (Non-existence of monotone equilibria in the second-price auction). Consider a second-price auction with two bidders and valuations uniformly distributed on $[0,1]$. Using equations (3) and (4) we get that

$$
\int_{\tau}^{v} \beta(x) d x=\int_{\tau}^{v} x d x+\mathcal{W}_{\tau}(v)-\mathcal{W}_{\tau}(\tau)
$$

which implies $\beta(v)=v+\mathcal{W}_{\tau}^{\prime}(v)$. In the second-price auction we have for $v \geq \tau$

$$
\mathcal{W}_{\tau}(v)=\tau \lambda \mathbb{E}(V \mid V \geq \tau)+\int_{\tau}^{v} \lambda \mathbb{E}(V \mid V \geq x) d x+(1-v) \lambda v
$$

Hence,

$$
\beta(v)=v+\mathcal{W}_{\tau}^{\prime}(v)=v+\lambda \frac{3}{2}(1-v)
$$

For $\lambda>2 / 3$ the bidding strategy is decreasing in $v$, hence an increasing equilibrium does not exist. Consequently, a second-price auction fails to allocate the good efficiently and is not optimal. With two bidders participation is automatically observable in a second-price auction, because the losing bidders participation decision can be inferred from the price. Hence, the non-existence result does not depend on whether participation is observable.

\section{Convex Signaling Concerns}

In this section, we consider convex signaling concerns, i.e., the case where $\Phi$ is strictly increasing and convex. With convex signaling concern it is no longer true that the total signaling value is independent of the auction design. Yet, any auction induces a distribution over posterior means that averages to the same mean, hence is a meanpreserving spread. With a convex $\Phi$ it is then optimal to 'disclose' as much information as possible, because this increases the signaling value. The maximal amount of information that an auction can disclose corresponds to fully disclosing the types of participating bidders, as no information from non-participating bidders is obtained.

Proposition 3 (Optimal auction under convexity). Consider a standard auction, in which every bidder follows a strictly increasing bidding strategy, and participates whenever his type is above $\tau$. The revenue in this auction is at most

$$
\operatorname{Rev}^{\mathcal{M}}(\tau)+n\left(\int_{\tau}^{\bar{v}} \Phi(v) d F(v)-(1-F(\tau)) \Phi(\mathbb{E}[V \mid V \leq \tau])\right)
$$

The all-pay auction exhibits the described equilibrium and attains the revenue bound. 
Proof. From Proposition 1 we have that revenue equals (5). Define $H_{\tau}^{\max }$ the distribution over posterior means as follows

$$
H_{\tau}^{\max }(v)= \begin{cases}0, & \text { if } \underline{v} \leq v<\mathbb{E}[V \mid V \leq \tau] \\ F(\tau), & \text { if } \mathbb{E}[V \mid V \leq \tau] \leq v \leq \tau \\ F(v), & \text { if } \tau<v \leq \bar{v}\end{cases}
$$

The distribution $H_{\tau}^{\max }$ has a mass point at $\mathbb{E}[V \mid V \leq \tau]$ and otherwise corresponds to the prior $F$ for values above $\tau$. $H_{\tau}^{\max }$ is the distribution over posterior beliefs induced by a rule that fully reveals the type of a participating bidder, and otherwise discloses no further information. Because the observer can never distinguish the types of nonparticipating bidders, we have that $H_{\tau}^{\max }$ is a mean-preserving spread of $H_{\tau}^{A}$. Convexity of $\Phi$ thus implies

$$
\int_{\underline{v}}^{\bar{v}} \Phi(v) d H_{\tau}^{A}(v) \leq \int_{\underline{v}}^{\bar{v}} \Phi(v) d H_{\tau}^{\max }(v)=F(\tau) \Phi(\mathbb{E}[V \mid V \leq \tau])+\int_{\tau}^{\bar{v}} \Phi(v) d F(v) .
$$

Together with our previous observation that $\mathcal{W}_{\tau, 0}^{A} \geq \Phi(\mathbb{E}[V \mid V \leq \tau])$ the revenue bound (8) follows.

To show the revenue bound (8) can be attained, consider an all-pay auction with an entry fee $\varphi:=G(\tau) \tau+\Phi(\tau)-\Phi(\mathbb{E}[V \mid V \leq \tau])$. We establish the following equilibrium. A bidder enters whenever $v \geq \tau$ and bids

$$
\beta(v)=\int_{\tau}^{v} x d G(x)+\Phi(v)-\Phi(\tau)
$$

Denote $\Pi(v, \widetilde{v})$ the expected utility of a type $v$ when mimicking the strategy of type $\widetilde{v}$. For $\widetilde{v} \geq \tau$ we have that

$$
\begin{aligned}
\Pi(v, \widetilde{v}) & =G(\widetilde{v}) v+\Phi(\widetilde{v})-\beta(\widetilde{v})-\varphi \\
& =G(\widetilde{v}) v-G(\tau) \tau-\int_{\tau}^{v} x d G(x)+\Phi(\mathbb{E}[V \mid V \leq \tau])
\end{aligned}
$$

Hence, for all $v, \widetilde{v} \geq \tau$ it follows that

$$
\Pi(v, v)-\Pi(v, \widetilde{v})=\int_{\widetilde{v}}^{v}(v-x) d G(x) \geq 0
$$

In addition, for all $v<\tau \leq \widetilde{v}$ we have that $\Pi(v, v)=\Phi(\mathbb{E}[V \mid V \leq \tau])>\Pi(v, \widetilde{v})$ and, similarly, $\Pi(\widetilde{v}, \widetilde{v})>\Phi(\mathbb{E}[V \mid V \leq \tau])$. The all-pay auction induces the distribution over posterior means $H_{\tau}^{\max }$. Using the first-part of the proof shows that the revenue coincides with (8). 
Part of our analysis of finding the optimal auction amounts to determining the optimal information structure. We maximize (5) over the set of distributions $H_{\tau}^{A}$ which are a mean-preserving spread of $H_{\tau}^{\max }$, where $H_{\tau}^{\max }$ is the distribution over posterior means of a disclosure policy that reveals the true type $v$ if $v \geq \tau$ and lumps all other types on one signal. Put differently, $H_{\tau}^{\max }$ arises from disclosing the (maximal) information gathered by running the auction. Dworczak and Martini (2019) provide a solution to this problem, which in our case of a convex signaling function $\Phi$ yields full disclosure. But notice an important difference to their analysis: the signaling value of non-participating bidders $\mathcal{W}_{\tau, 0}$ negatively enters the auctioneer's objective. In the convex case, maximizing $\int_{\underline{v}}^{o v} \Phi(v) \mathrm{d} H(v)$ coincidentally minimizes $\mathcal{W}_{\tau, 0}^{A}$ and hence the solution obtains.

Proposition 3 shows that the all-pay auction yields maximal revenue when the bidders' signaling concerns are convex. This is not a property of the payment rule in an all-pay auction, but a consequence of the induced revelation of bidders' types. In general, full disclosure leads to a separation of bidding and signaling incentives, and any auction achieves the upper bound on revenue when the entire vector of bids gets disclosed. More precisely, any standard auction $A$ that features a strictly monotone equilibrium if there are no signaling concerns, maintains a strictly monotone equilibrium in the presence of signaling concerns, provided that all bids are public. Equilibrium strategies for the auction with signaling can be constructed from their counterparts without signaling via the formula $\beta(v)=\beta^{\mathcal{M}}(v)+\Phi(v)-\Phi(\mathbb{E}[V \mid V \leq \tau])$, where $\beta^{\mathcal{M}}$ denotes the bidding strategy for the respective auction without signaling concerns.

Corollary 2 (Revenue Equivalence with Full Bid Disclosure). Consider a standard auction, in which every bidder follows a strictly increasing bidding strategy, and participates whenever his type is above $\tau$. If the auction discloses all bids, revenue coincides with (8).

We derive the equilibrium of the first-price auction in the following Example.

Example 3 (First-price auction with full bid disclosure). Reconsider the first-price auction as in Example 1, but now with a convex signaling value $\Phi(\cdot)$. To induce the participation cut-off $\tau$ the auctioneer sets the reserve bid to $r(\tau)=\tau+\Phi(\tau) / G(\tau)-\Phi(\mathbb{E}[V \mid V \leq$ $\tau]) / G(\tau)$. The auction gives rise to an equilibrium where bidders use the following increasing bidding strategy

$$
\beta(v)= \begin{cases}0, & \underline{v} \leq v<\tau \\ \frac{G(v) v-G(\tau) \tau}{G(v)}+\int_{\tau}^{v} x d \frac{G(x)}{G(v)}+\frac{\Phi(v)}{G(v)}-\frac{\Phi(\mathbb{E}[V \mid V \leq \tau])}{G(v)}, & \tau \leq v \leq \bar{v}\end{cases}
$$

As in the case of linear signaling concerns, we can look for the optimal participation threshold $\tau^{\star}$ in the auction. The bidders' signaling concerns induce the auctioneer to reduce the threshold for participation below the optimal level without signaling. 
Corollary 3. Assume virtual valuations are increasing. In the revenue maximizing auction more bidders participate than if bidders had no signaling concern, i.e. $\tau^{\star}<\tau^{\mathcal{M}}$.

Proof. It follows similar steps as the proof of Corollary 1.

Remark 2. Under convex (and linear) signaling it is also possible to show that auctions maximize the seller's revenue across all mechanisms. To see this, assume we were to analyze direct mechanisms that in addition disclose a signal for each bidder (as in Dworczak (2020)). To simplify, assume in addition that transfers are unobservable to the receiver. Similar to (2), we can prove a payoff-equivalence and decompose revenue in a non-signaling and a signaling component. The signaling component is maximal for full disclosure of participating types (or any disclosure plus observable participation in the linear case). Under the usual regularity condition, the non-signaling component is maximized by allocating the good to the bidder with the highest value above a reserve value. Note that full disclosure of participating types can be achieved, e.g., via disclosing a bidder's interim payment.

\section{Concave Signaling Concerns}

In this section, we consider concave signaling concerns. From Proposition 1 we have that the revenue can be decomposed as in (5). Information disclosure directly affects the second and third components, respectively $\int \Phi(v) \mathrm{d} H_{\tau}^{A}(v)$ and $\mathcal{W}_{\tau, 0}^{A}$. Under concavity, the signaling value $\int \Phi(v) \mathrm{d} H_{\tau}^{A}(v)$ increases if the auction discloses fewer information about bidders. At the same time, revealing less information increases the outside option, i.e., the signaling value of non-participating bidders $\mathcal{W}_{\tau, 0}^{A}$.

More precisely, consider first the signaling value, given by the term $\int \Phi(v) \mathrm{d} H_{\tau}^{A}(v)$. Information disclosure amounts to choosing a distribution over posterior means $H_{\tau}^{A}$, where $H_{\tau}^{A}$ is a mean-preserving spread of the minimal information distribution $H_{\tau}^{\mathrm{min}}$. The latter distribution is given by the policy that discloses only the winner's identity, if the auction has a winner. Because the inference function $\Phi$ is concave, the signaling value is maximal for $H_{\tau}^{A}=H_{\tau}^{\text {min }}$. In words, the signaling value is maximal when the auction reveals no additional information beyond the winner's identity (which has to be revealed by assumption). Next consider the term $\mathcal{W}_{\tau, 0}^{A}$, the expected inference of a bidder who does not participate. As before, $\mathcal{W}_{\tau, 0}^{A}$ is minimal if the auction reveals whether a bidder participated, for instance via charging an entry fee. In contrast to the convex case, maximizing the signaling value and minimizing $\mathcal{W}_{\tau, 0}^{A}$ conflict with each other. In particular, revealing whether a bidder participated in the auction reveals more information than only revealing the winner's identity. In general this yields a non-trivial trade-off without a straightforward solution. 
Define $H_{\tau}^{P}$ the distribution over posterior means that arises from a disclosure policy which reveals the winner's identity and whether a bidder participated in an auction with participation threshold $\tau$. Note that $H_{\underline{v}}^{P}=H_{\underline{v}}^{\min }$. Moreover, define

$$
\operatorname{Rev}^{P}(\tau)=\operatorname{Rev}^{\mathcal{M}}(\tau)+n\left(\int_{\underline{v}}^{\bar{v}} \Phi(v) \mathrm{d} H_{\tau}^{P}(v)-\mathbb{E}[V \mid V<\tau]\right) .
$$

Proposition 4. Consider a standard auction, in which every bidder follows a strictly increasing bidding strategy, and participates whenever his type is above $\tau$.

(i) If participation is fully observable we have that $\operatorname{Rev}(\tau) \leq \operatorname{Rev}^{P}(\tau)$.

(ii) There exists $\tau^{\prime}>\underline{v}$ such that $\operatorname{Rev}(\tau) \leq \operatorname{Rev}^{P}(\tau)$ whenever $\tau<\tau^{\prime}$.

Proof. From Proposition 1 we have that revenue equals (5). With observable participation we have $\mathcal{W}_{\tau, 0}^{A}=\mathbb{E}[V \mid V<\tau]$, independent of the specific auction format. Furthermore, because $\Phi$ is concave the signaling value $\int_{\underline{v}}^{\bar{v}} \Phi(v) \mathrm{d} H_{\tau}^{A}(v)$ is maximal when only the winner's identity is disclosed in addition, i.e., when $H_{\tau}^{A}=H_{\tau}^{P}$. This proves (i).

To prove (ii), note that for every auction in which participation is not fully observable we have $\mathcal{W}_{\tau, 0}^{A}>\mathbb{E}[V \mid V<\tau]$. Moreover, for $\tau=\underline{v}$ we have $\int_{\underline{v}}^{\bar{v}} \Phi(v) \mathrm{d} H_{\underline{v}}^{A}(v) \leq$ $\int_{\underline{v}}^{\bar{v}} \Phi(v) \mathrm{d} H^{\min }(v)$ by concavity of $\Phi$. Hence, $\operatorname{Rev}(\underline{v})<\operatorname{Rev}^{P}(\underline{v})$. Both $\overline{\mathcal{W}}_{\tau, 0}^{A}$ and $\int_{\underline{v}}^{\bar{v}} \Phi(v) \mathrm{d} H_{\tau}^{A}(v)$ are continuous in the participation threshold $\tau$, hence (ii) follows by continuity from the previous assertion.

Proposition 4 derives an upper bound for the revenue if either participation is fully observable, or many bidder types participate. In other cases, i.e., when few bidder types participate in the auction, other information disclosure policies than revealing the winner's identity and whether a bidder participated may lead to higher revenue. In particular this may require fewer information to be provided, i.e., not concealing participation decisions.

The bound derived in Proposition 4 stems from a (hypothetical) auction that discloses only the winner's identity, and a list of participating bidders. However to guarantee existence of a separating equilibrium the auction necessarily uses a discriminatory payment rule, which ultimately reveals information beyond the winner's identity. To see this, note that if we had only payments for winner and loser's, there would not exist an equilibrium with strictly increasing bidding strategies. This suggests the revenue bound is too large. Proposition 5 shows that the revenue bound is indeed tight. The intuition behind the results is as follows. The payment rule has to be discriminatory, as explained above. But only the interim expected payment is crucial, not how payments realize ex-post. ${ }^{15}$ In

\footnotetext{
${ }^{15}$ This is also the key observation behind payoff-equivalence in auctions and mechanism design.
} 
fact, it is sufficient if the winner makes a payment with arbitrarily small probability, provided that this payment is large such that the interim expected payment is the same. If payments are infrequent, the outside observers additional inference (beyond the winner's identity) has little impact on the signaling value.

Proposition 5. For every $\varepsilon>0$ and every $\tau$ there is an auction that exhibits an equilibrium with strictly increasing bidding strategies, for which $\operatorname{Rev}(\tau)>\operatorname{Rev}^{P}(\tau)-\varepsilon$.

Proof. Consider the following variant of a first-price auction: Bidders submit non-negative bids, the bidder submitting the highest bid wins and with exogenous probability $1-\varepsilon$ makes no payment, but pays his own bid with probability $\varepsilon .{ }^{16}$ Every bidder has to pay the entry fee $\varphi$ before submitting a bid. The expected profit of a bidder of type $v$ upon entering the auction and bidding as if he was type $v^{\prime}$ is

$$
\Pi\left(v \mid v^{\prime}\right)=G\left(v^{\prime}\right)\left(v-\varepsilon \beta\left(v^{\prime}\right)\right)+\mathcal{W}_{\tau}\left(v^{\prime}\right)-\varphi
$$

From the first-order condition we get

$$
\beta^{\star}(v)=\frac{1}{\varepsilon} \beta^{\mathcal{M}}(v)+\frac{\mathcal{W}_{\tau}(v)-\mathcal{W}_{\tau}(\tau)}{\varepsilon G(v)},
$$

where $\beta^{\mathcal{M}}$ is the bidding strategy in a first-price auction without signaling and entry fee that induces only types above $\tau$ to participate. Note that we have used the fact that $\beta(\tau)=0$, which is true because in equilibrium type $\tau$ only wins the auction when no other bidder enters and is thus not willing to bid a strictly positive amount. Furthermore, to induce participation for all types above $\tau$ the fee has to satisfy

$$
\mathcal{W}_{\tau, 0}=G(\tau) \tau+\mathcal{W}_{\tau}(\tau)-\varphi \quad \Leftrightarrow \quad \varphi=G(\tau) \tau+\mathcal{W}_{\tau}(\tau)-\mathcal{W}_{\tau, 0}
$$

The revenue is thus given by

$$
\begin{aligned}
\operatorname{Rev}^{\varepsilon}(\tau) & =n(1-F(\tau)) \varphi+\left(1-F^{n}(\tau)\right) \mathbb{E}\left[\varepsilon \beta^{\star}\left(V_{1}\right) \mid V_{1} \geq \tau\right] \\
& =n(1-F(\tau))\left(G(\tau) \tau+\mathcal{W}_{\tau}(\tau)-\mathcal{W}_{\tau, 0}\right)+\int_{\tau}^{\bar{v}}\left(\beta^{\mathcal{M}}(s)+\frac{\mathcal{W}_{\tau}(s)-\mathcal{W}_{\tau}(\tau)}{G(s)}\right) \mathrm{d} F^{n}(s) \\
& =\operatorname{Rev}^{\mathcal{M}}(\tau)+n(1-F(\tau))\left(\mathcal{W}_{\tau}(\tau)-\mathcal{W}_{\tau, 0}\right)+n \int_{\tau}^{\bar{v}}\left(\mathcal{W}_{\tau}(s)-\mathcal{W}_{\tau}(\tau)\right) f(s) \mathrm{d} s \\
& =\operatorname{Rev}^{\mathcal{M}}(\tau)+n\left[F(\tau) \mathcal{W}_{\tau, 0}+\int_{\tau}^{\bar{v}} \mathcal{W}_{\tau}(s) f(s) \mathrm{d} s-\mathcal{W}_{\tau, 0}\right]
\end{aligned}
$$

\footnotetext{
${ }^{16}$ The superscript $A$ is omitted in the proof, as it goes through a specific first-price auction.
} 
Note that

$$
\begin{aligned}
\mathcal{W}_{\tau}(s)= & \sum_{k=1}^{n} \mathcal{B}_{n-1, F(\tau)}(k-1)\left\{F_{k}(s \mid \tau) \cdot\left(\varepsilon \Phi(s)+(1-\varepsilon) \Phi\left(v_{W, k}\right)\right)\right. \\
& \left.+\left(1-F_{k}(s \mid \tau)\right) \cdot\left(\varepsilon \int_{s}^{\bar{v}} \Phi\left(v_{L, k}(x)\right) \mathrm{d} F_{k}(x \mid \tau)+(1-\varepsilon) \Phi\left(v_{L, k}\right)\right)\right\} \\
= & \sum_{k=1}^{n} \mathcal{B}_{n-1, F(\tau)}(k-1)\left\{F_{k}(s \mid \tau) \Phi\left(v_{W, k}\right)+\left(1-F_{k}(s \mid \tau)\right) \Phi\left(v_{L, k}\right)\right\} \\
-\varepsilon & \sum_{k=1}^{n} \mathcal{B}_{n-1, F(\tau)}(k-1)\left\{F_{k}(s \mid \tau)\left(\Phi(s)-\Phi\left(v_{W, k}\right)\right)\right. \\
& \left.+\left(1-F_{k}(s \mid \tau)\right)\left(\int_{s}^{\bar{v}} \Phi\left(v_{L, k}(x)\right) \mathrm{d} F_{k}(x \mid \tau)-\Phi\left(v_{L, k}\right)\right)\right\}
\end{aligned}
$$

where $\mathcal{B}_{n-1, F(\tau)}(k-1):=\left(\begin{array}{l}n-1 \\ k-1\end{array}\right) F(\tau)^{n-k}(1-F(\tau))^{k-1}, v_{W, k}:=\mathbb{E}\left[V_{1} \mid V_{k} \geq \tau>V_{k+1}\right]$, $v_{L, k}:=\mathbb{E}\left[V \mid V_{1}>V \geq V_{k} \geq \tau>V_{k+1}\right], v_{L, k}(s):=\mathbb{E}\left[V \mid V_{1}=s, s>V \geq V_{k} \geq \tau>V_{k+1}\right]$ and $F_{k}(v \mid \tau):=\left(\frac{F(v)-F(\tau)}{1-F(\tau)}\right)^{k-1}$ for all $k=1, \ldots, n$ denotes the conditional probability of the maximum of the $k-1$ other bids if all of these exceed $\tau$. Hence,

$$
\begin{aligned}
\int_{\tau}^{\bar{v}} \mathcal{W}_{\tau}(s) f(s) \mathrm{d} s= & \sum_{k=1}^{n} \mathcal{B}_{n-1, F(\tau)}(k-1)\left\{\frac{1}{k} \Phi\left(v_{W, k}\right)+\frac{k-1}{k} \Phi\left(v_{L, k}\right)\right\} \\
-\varepsilon & \sum_{k=1}^{n} \mathcal{B}_{n-1, F(\tau)}(k-1)\left\{\int_{\tau}^{\bar{v}} F_{k}(s \mid \tau)\left(\Phi(s)-\Phi\left(v_{W, k}\right)\right) f(s) \mathrm{d} s\right. \\
& \left.+\int_{\tau}^{\bar{v}}\left(1-F_{k}(s \mid \tau)\right)\left(\int_{s}^{\bar{v}} \Phi\left(v_{L, k}(x)\right) \mathrm{d} F_{k}(x \mid \tau)-\Phi\left(v_{L, k}\right)\right) f(s) \mathrm{d} s\right\} \\
= & \sum_{k=1}^{n} \mathcal{B}_{n-1, F(\tau)}(k-1)\left\{\frac{1}{k} \Phi\left(v_{W, k}\right)+\frac{k-1}{k} \Phi\left(v_{L, k}\right)\right\}-\varepsilon C,
\end{aligned}
$$

where by concavity of $\Phi$ and compactness of the support of the bidders' valuations we have $C>0$ and finite. Plugging the above expression back into (12) and noting that $\mathcal{W}_{\tau, 0}=\mathbb{E}[V \mid V<\tau]$ (because participation is observable) yields $\operatorname{Rev}^{\varepsilon}(\tau)=\operatorname{Rev}^{P}(\tau)-$ $\varepsilon C \rightarrow \operatorname{Rev}^{P}(\tau)$ as $\varepsilon \rightarrow 0$.

Propositions 4 and 5 reveal a fundamental difference between pure information design and mechanism design with information disclosure. In information design the sender has costless access to all information, while in mechanism design the information is privately held by the agents. In our setting the auctioneer benefits from revealing additional information, namely whether a bidder participated. Such disclosure reduces the value of the bidders' outside option, i.e., the expected signaling value from non-participation. The reduced outside option allows the auctioneer to extract more revenue from bidders. The benefit from revealing the bidders' participation is larger, the lower the participation threshold $\tau$. For $\tau \approx \underline{v}$ it becomes optimal to disclose only the winner's identity and all 
participation decisions.

Remark 3. With concave signaling concerns an auction does not necessarily constitute an optimal mechanism, hence our focus on equilibria in strictly increasing strategies is restrictive. To see this let us consider the following example with 2 bidders, valuations drawn from a uniform distribution on $[1,2]$, and a signaling function $\Phi(v)=k \sqrt{v}$ with $k>0$. Therefore, the optimal auction uses no reserve price, and participation is fully observable. Following Propositions 4 and 5, the maximal revenue of an auction is then

$$
R e v^{A}=\frac{4}{3}+2 k\left(\frac{1}{2} \sqrt{\frac{5}{3}}+\frac{1}{2} \sqrt{\frac{4}{3}}\right) .
$$

Now consider a lottery, in which the object is allocated at random. Provided that both bidders participate, type $v$ 's expected utility is $\frac{1}{2} v+k \sqrt{\frac{1}{2}}$. Note that the allocation, i.e., who is assigned the object, does not reveal new information about the bidders' types. To ensure full participation the seller can charge a participation fee of $\frac{1}{2}+k \sqrt{\frac{3}{2}}$, thus revenue is

$$
R e v^{P}=1+2 k \sqrt{\frac{3}{2}}
$$

Clearly, for sufficiently large $k$ we have $R e v^{P}>\operatorname{Rev} v^{A} \cdot{ }^{17}$ Recall that we assume the final allocation (i.e., who gets the object) is observable. Therefore, a mechanism that implements an allocation rule that conditions on reported types necessarily reveals information about bidders. With a concave signaling function, information revealed via the allocation reduces the signaling value the auctioneer can extract. If signaling concerns are sufficiently strong, the gain in signaling value outweighs the loss in terms of standard revenue $\operatorname{Rev}^{\mathcal{M}}$, hence an auction is no longer optimal. Whether a lottery represents an optimal mechanism depends on the marginal gain from improving the allocation versus the marginal loss in terms of signaling value this brings about. ${ }^{18}$

\section{Discussion}

In this paper, we analyze optimal auctions in an independent private values environment with signaling, i.e. where bidders' care about the perception of a third party. To keep the analysis concise and tractable we focused on linear, convex and concave signaling concerns. The results of Dworczak and Martini (2019) indicate that the disclosure policy maximizing the signaling value for general preferences is a combination of intervals where the type is fully disclosed and intervals on which types are fully pooled. However, it is

\footnotetext{
${ }^{17}$ Straightforward computations lead to a threshold of $\hat{k} \approx 87.84$. This threshold would reduce with either more bidders, more concave signaling function $\Phi$, or a more spread-out distribution.

${ }^{18}$ However, note that a formal analysis is all but straightforward. The presence of signaling prevents us from using standard methods, such as pointwise maximization of the objective.
} 
not straightforward to translate such a disclosure rule into a payment rule for a standard auction. Understanding the polar cases of convexity and concavity allows us to address a preference for the aftermarket that has been studied in the literature on information design, namely where $\Phi$ is a distribution function. ${ }^{19}$ Under regularity conditions, there is a unique value $\hat{v}$ such that $\Phi$ is convex on $[\underline{v}, \hat{v}]$ and concave on $[\hat{v}, \bar{v}]$. Hence, if the participation threshold is sufficiently high we are back in the concave case. Otherwise, maximizing revenue calls for revealing low bids while at the same time pooling higher bids. Disclosure of low value implies that the auctioneer again prefers to disclose whether a bidder participated.

A natural follow-up question concerns the extent to which our results can be generalized to a richer class of mechanisms. Beyond mechanism design, that could provide new and exciting perspectives in applied fields such as advertising, marketing science and industrial organization. For instance, the literature on conspicuous consumption (e.g., Bagwell and Bernheim (1996) and Corneo and Jeanne (1997)) studies product markets where the consumption value depends on the belief of a social contact. A profitmaximizing seller will try to exploit this by tailoring its product line and prices to the information revealed by the consumer's choice. That will lead to new insights about consumer behavior and firm strategies that exploit signaling concerns. ${ }^{20}$

\section{References}

Bagwell, L. S. and Bernheim, B. D. (1996), 'Veblen effects in a theory of conspicuous consumption', American Economic Review 86(3), 349-73.

Bos, O. and Truyts, T. (2018), Entry in first-price auctions with signaling. Mimeo.

Bos, O. and Truyts, T. (2019), Auctions with signaling concerns. Mimeo.

Calzolari, G. and Pavan, A. (2006a), 'Monopoly with resale', RAND Journal of Economics 37(2), 362-375.

Calzolari, G. and Pavan, A. (2006b), 'On the optimality of privacy in sequential contracting', Journal of Economic Theory 130, 168-204.

Corneo, G. and Jeanne, O. (1997), 'Conspicuous consumption, snobbism and conformism', Journal of Public Economics 66(1), 55-71.

\footnotetext{
${ }^{19}$ Rayo and Segal (2010) study such a sender-receiver game. The receiver chooses a binary action $a \in\{0,1\}$. Choosing $a=0$ yields a fixed utility $r$ which is distributed according to some distribution function $G$. Choosing $a=1$ yields utility $\theta$, where $\theta$ is the sender's private information. The sender wants to maximize the probability of choosing $a=1$. Hence, the sender's reduced form utility is $G(\mathbb{E}(\theta))$.

${ }^{20}$ See Rayo and Segal (2010) and Friedrichsen (2018) for analyses into that direction.
} 
Das Varma, G. (2003), 'Bidding for a process innovation under alternative modes of competition', International Journal of Industrial Organization 21(1), 15-37.

Dworczak, P. (2020), 'Mechanism design with aftermarkets: Cutoff mechanisms', Econometrica (forthcoming) .

Dworczak, P. and Martini, G. (2019), 'The simple economics of optimal persuasion', Journal of Political Economy 127(5), 1993-2048.

Friedrichsen, J. (2018), Signals sell: Product lines when consumers differ both in taste for quality and image concern, Technical report, CRC TRR 190 Rationality and Competition.

Giovannoni, F. and Makris, M. (2014), 'Reputational bidding', International Economic Review 55(3), 693-710.

Goeree, J. K. (2003), 'Bidding for the future: Signaling in auctions with an aftermarket', Journal of Economic Theory 108(2), 345-364.

Jehiel, P. and Moldovanu, B. (2000), 'Auctions with downstream interaction among buyers', RAND Journal of Economics 31(4), 768-791.

Jehiel, P. and Moldovanu, B. (2001), 'Efficient design with interdependent valuations', Econometrica 69(5), 1237-1259.

Katzman, B. E. and Rhodes-Kropf, M. (2008), 'The consequences of information revealed in auctions', Applied Economics Research Bulletin 2, 53-87.

Krishna, V. (2009), Auction Theory, second edn, Academic Press, Elsevier.

Liu, T. (2012), 'Takeover bidding with signaling incentives', The Review of Financial Studies 25(2), 522-556.

Lizzeri, A. (1999), 'Information revelation and certification intermediaries', The RAND Journal of Economics 30(2), 214-231.

Mandel, B. R. (2009), 'Art as an investment and conspicuous consumption good', American Economic Review 99(4), 1653-63.

Milgrom, P. and Segal, I. (2002), 'Envelope theorems for arbitrary choice sets', Econometrica 70(2), 583-601.

Milgrom, P. and Weber, R. (1982), 'A theory of auctions and competitive bidding', Econometrica 50(5), 1089-1122. 
Molnar, J. and Virag, G. (2008), 'Revenue maximizing auctions with market interaction and signaling', Economics Letters 99(2), 360-363.

Myerson, R. (1981), 'Optimal auction design', Mathematics of Operations Research 6(1), 58-73.

Rayo, L. and Segal, I. (2010), 'Optimal information disclosure', Journal of Political Economy 118(5), 949-987.

Riley, J. G. and Samuelson, W. F. (1981), 'Optimal auctions', American Economic Review 71(3), 381-392.

von Scarpatetti, B. and Wasser, C. (2010), Signaling in auctions among competitors. Mimeo.

Wan, Z. and Beil, D. R. (2009), 'Rfq auctions with supplier qualification screening', Operations Research 57(4), 934-949. 
Download ZEW Discussion Papers from our ftp server:

http://ftp.zew.de/pub/zew-docs/dp/

or see:

https://www.ssrn.com/link/ZEW-Ctr-Euro-Econ-Research.html

https://ideas.repec.org/s/zbw/zewdip.html

$$
\text { // }
$$

IMPRINT

ZEW - Leibniz-Zentrum für Europäische Wirtschaftsforschung GmbH Mannheim

ZEW - Leibniz Centre for European

Economic Research

L 7,1 68161 Mannheim · Germany

Phone +49621 1235-01

info@zew.de·zew.de

Discussion Papers are intended to make results of ZEW research promptly available to other economists in order to encourage discussion and suggestions for revisions. The authors are solely responsible for the contents which do not necessarily represent the opinion of the ZEW. 